\title{
Growth, mineral nutrient content and nitrogen metabo- lism in Laccaria laccata-inoculated and non-inoculated Douglas fir seedlings during their growth period
}

\author{
M. Chalot, B. Botton and J. Banvoy \\ Université de Nancy I, Faculté des Sciences, Laboratoire de Physiologie Végétale et Forestière, \\ BP 239, 54506 Vandouvre-lès-Nancy, France
}

\section{Introduction}

Enhanced plant growth caused by ectomycorrhizal inoculation in the nursery is well documented: thus Laccaria strains have proven to be very efficient for improving growth of various conifers (Le Tacon and Bouchard, 1986). The investigations described herein were carried out to obtain information on physiological and biochemical modifications that can explain such stimulation. The present study compared the seasonal changes in protein and amino acid levels, aspartate aminotransferase activity and nutrient content in both L. laccata-inoculated and non-inoculated Douglas fir grown in a forest nursery.

\section{Materials and Methods}

After $1 \mathrm{yr}$ of growth in the nursery, 12 seedlings from both untreated and treated plots were collected at random, bimonthly from May to September. Root and top fresh weights and heights were measured for each seedling. Parts of the needles and roots were removed immediately after collection for amino acid, protein and enzyme determinations. Aspartate aminotransferase (AAT) activity was determined spectrophotometrically and proteins were measured using a colorimetric method. Amino acids were analyzed using an HPLC procedure. Total $\mathrm{N}$ was measured by a microKjeldahl method, total $\mathrm{P}$ by a colorimetric method and $\mathrm{K}$ and $\mathrm{Mg}$ by atomic absorption spectrophotometry.

\section{Results}

By the end of the growing period, L. laccata-inoculated seedlings were significantly higher (not shown) and had accumulated more than 2-fold the fresh matter of the controls (Table I). In expanding shoots, N, $\mathrm{P}, \mathrm{K}$ and $\mathrm{Mg}$ concentrations decreased rapidly until mid-August (Fig. $1 \mathrm{~A}, \mathrm{~B}, \mathrm{C}, \mathrm{D}$ ) and tended to increase after growth ceased. No significant difference was found in nutrient analysis of stems and roots between treated and untreated plants (not shown). By contrast, statistically significant (ANOVA, $P=0.05$ ) changes were observed for leaf nutrient analysis 
Table I. Seasonal variations of fresh matter ( $g / p l a n t \pm S D$ ) in $L$. laccata-inoculated and non-inoculated (control) Douglas fir, 1 yr old at the beginning of the experiment.

\begin{tabular}{lrrrr}
\hline & \multicolumn{3}{l}{ Date of sampling } & \\
\cline { 2 - 5 } & May 11 & June 15 & \multicolumn{1}{c}{ July 20 } & \multicolumn{1}{c}{ Sept. 8 } \\
\hline Non-inoculated controls & $0.6 \pm 0.3$ & $4.2 \pm 1.3$ & $7.1 \pm 1.6$ & $7.9 \pm 1.3$ \\
L. laccata-inoculated fir & $4.6 \pm 1.2$ & $11.2 \pm 2.1$ & $16.3 \pm 2.3$ & $16.8 \pm 2.7$ \\
\hline
\end{tabular}
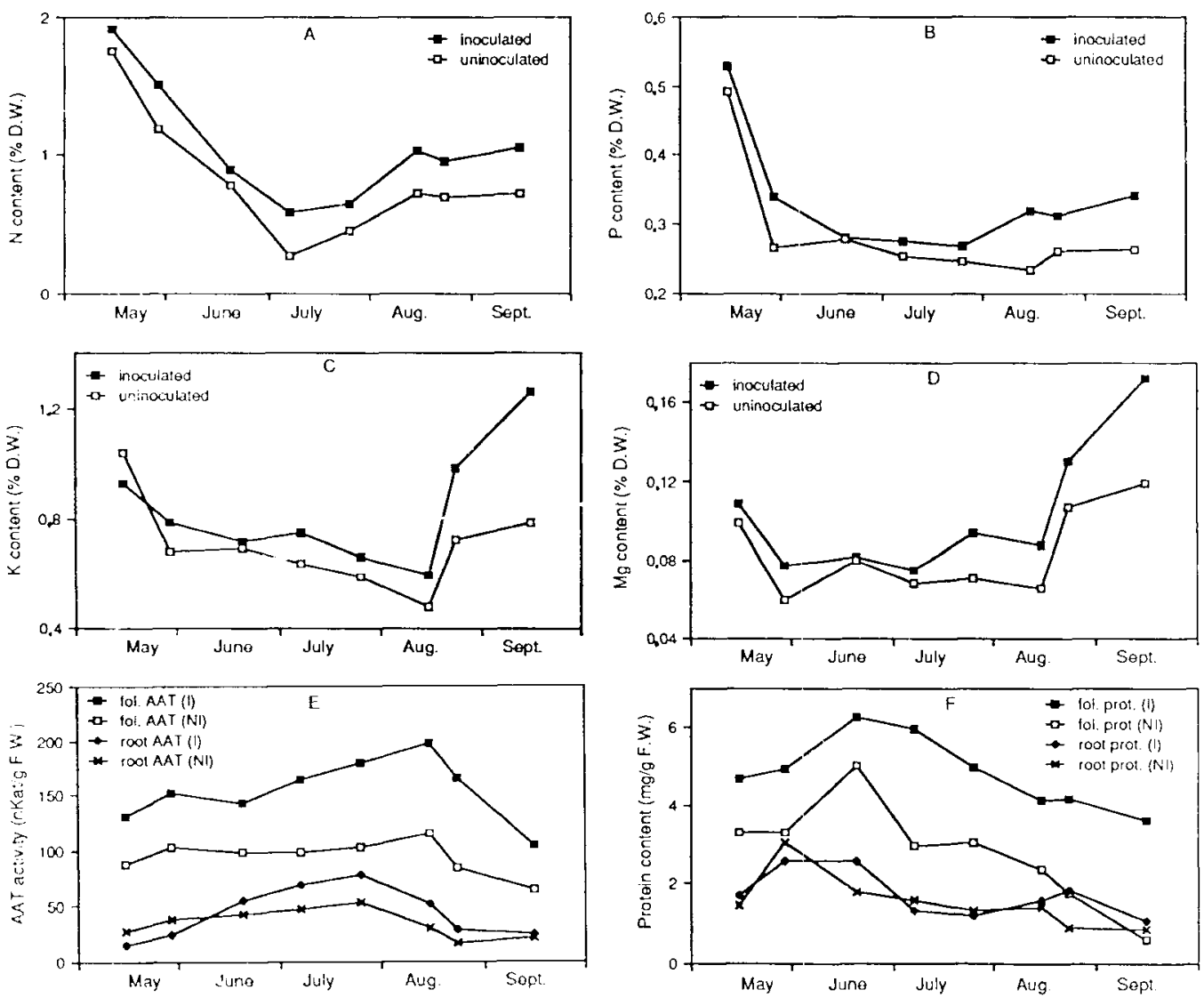

Fig. 1. Seasonal changes in nutrient and protein contents. AAT activity of $L$. laccata-inoculated or non-inoculated Douglas fir seedlings grown in a forest nursery. (fol: foliar; I: inoculated; NI: non-inoculated). A: leaf nitrogen; B: leaf phosphorus; C: leaf potassium; D: leaf magnesium; E: leaf and root AAT activities; F: leaf and root proteins.

between treatments and, more particularly, by the end of the growing season (Fig. 1A, $B, C, D)$.
Concerning protein amounts and AAT activity levels, high and significant differences (ANOVA, $P=0.05$ ) were found in 


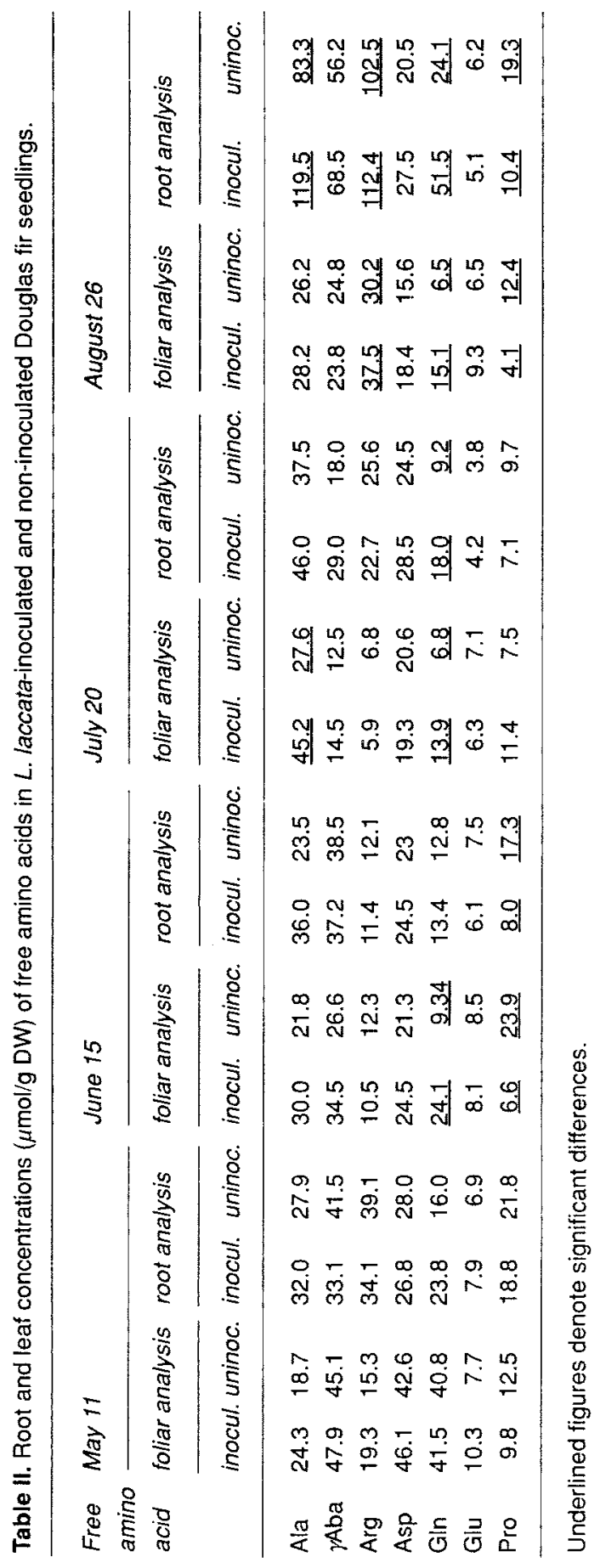


foliar analysis between inoculated seedlings and controls: leaves of inoculated Douglas fir accumulated higher protein amounts (Fig. 1F) and showed greater AAT capacities (Fig. 1E). By contrast, there was no difference in root analysis between treatments (Fig. 1E, F).

Alanine, aminobutyric acid, glutamine, aspartic acid, arginine, proline and glutamic acid were the major components of the free amino acid pools in root and leaf tissues. Alanine and glutamine in both root and leaf tissues appeared to be in higher amounts in L. laccata-inoculated Douglas fir, whereas proline preferentially accumulated in non-inoculated seedlings (Table II).

\section{Discussion and Conclusion}

Growth measurements reaffirmed the wellestablished importance of L. laccata strain 238 for improving seedling growth of Douglas fir (Le Tacon and Bouchard, 1986). Leaf nutrient content following inoculation also confirmed the importance of ectomycorrhizae for nutrient uptake, as reviewed by Harley (1969).

Our results also suggested that inoculations caused changes in the biochemical processes of the host tissues as demonstrated earlier (Nemec and Meredith, 1981; Krishna and Bagyara, 1983). Thus, alanine, proline and glutamine contents, protein amounts and AAT activity levels differed between controls and treated plants.
As a hypothesis, we might propose that lower AAT activity combined with a higher proline amount in non-inoculated Douglas fir characterized seedlings in stress-inducing situations. AAT is often considered as an indicator of physiological activity in plants affected by various internal or external factors (Cooper and Hill-Cottingham, 1974) and proline accumulation described as a response to environmental stress factors (Bode et al., 1985). Inoculation with efficient mycorrhizal strains might provide a way to suppress or at least to attenuate stress-inducing situations.

\section{References}

Bode J., Kuhn H.P. \& Wild A. (1985) The accumulation of proline in needles of damaged spruce. Forstwiss. Centralbl. 104, 353-360

Cooper D.R. \& Hill-Cottingham D.G. (1974) Glutamic dehydrogenase and glutamic oxaloacetate transaminase in apple tree. Physiol. Plant. 31, 193-199

Harley J.L. (1969) in: The Biology of Mycorrhizae. Leonard Hill, London

Krishna K.R. \& Bagyara D.J. (1983) Changes in the free amino nitrogen and protein fractions of groundnut caused by inoculation with VA mycorrhizas. Ann. Bot. 51, 399-401

Le Tacon F. \& Bouchard D. (1986) Effects of different ectomycorrhizal fungi on growth of larch, Douglas fir, Scots pine and Norway spruce seedlings in fumigated nursery soil. Acta Oecol. Appl. 7, 389-402

Nemec S. \& Meredith F. (1981) Amino acid content of leaves in mycorrhizal and non mycorrhizal citrus root stocks. Ann. Bot. 47, 203-222 